\title{
Quality of Life and Marilyn Monroe: Which Language Do They Speak?
}

\author{
Francesco De Blasio \\ Section of Pulmonary Rehabilitation, Private Hospital, Clinic Center, Naples, Italy
}

Since the 1970s, pulmonary rehabilitation (PR) has gradually become an important branch of respirology, in that it plays a more and more increasing role in the treatment of patients suffering from a variety of chronic and disabling diseases, among which chronic obstructive pulmonary disease (COPD) is the major one.

As soon as PR became one of the most utilized treatment options among chest-related specialists, it urged the need for outcome identifications and measurements. Fortunately, in the last decade we assisted at an overwhelming scientific production focusing upon PR. Moreover, in recent years major international scientific societies of pulmonology (i.e. the American Thoracic Society, the European Respiratory Society and the American College of Chest Physicians) produced interesting reports that have helped chest physicians to a more accurate approach. In 1997, the American College of Chest Physicians, jointly with the American Academy of Cardiovascular and Pulmonary Rehabilitation, first published evidence-based guidelines on PR defining its components and outcomes [1]. Amongst the latter there are dyspnea, employment of health care resources, survival and quality of life (QOL). The highest grade of scientific evidence (grade A) was given to dyspnea, with the recommendation that 'PR improves the symptoms of dyspnea in patients with COPD, whereas QOL received grade B with the same recommendation ('PR improves health-related quality of life in patients with COPD'). Subsequently, several excellent papers focusing on QOL modifications in patients undergoing PR protocols were published, justifying the proposal of a higher grade of scientific evidence [2].

Hence, QOL is one of the most compelling determinations attempted in evaluating PR, and recent efforts have been made toward either understanding quality from the patient's point of view or anchoring QOL to the health status [3]. To such an extent, excellent results have been obtained measuring QOL with disease-specific questionnaires, such as the Chronic Respiratory Questionnaire [4], the St. George's Respiratory Questionnaire [5] and the Maugeri Foundation Respiratory Failure Quesionnaire (MRF28) [6]. All these questionnaires represent validated tools which are widely used in clinical practice as well as in research.

Among the above-mentioned questionnaires, MRF28 has some specific peculiarities. First, it was designed for a specific group of patients, those who suffer from chronic respiratory failure, regardless of the underlying disease (e.g. COPD or chest wall abnormalities). Secondly, among its components, a unique feature is represented by sections on cognitive function and invalidity, the first relating to the effect of impaired cognitive function on daily life, the second including experience of social isolation and dependency on others.

\section{KARGER \\ Fax +41613061234 \\ E-Mail karger@karger.ch \\ www.karger.com \\ (C) 2004 S. Karger AG, Basel \\ 0025-7931/04/0716-0551\$21.00/0 \\ Accessible online at: www. karger.com/res}

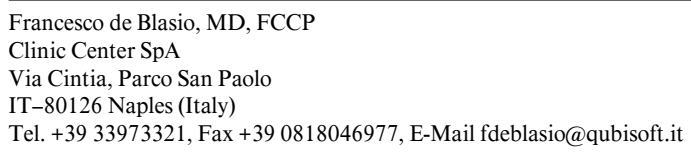


Originally presented in English, the questionnaire has been translated into different languages to be used in a multicenter multinational study with the aim of evaluating its validity and usefulness [7]. Of course, since we deal with a self-administered questionnaire, translation is extremely useful for its current utilization among patients speaking their own mother language. In other words, administering the English and the Italian version of the questionnaire to a homogeneous population of COPD patients, from London and Naples, for example, we might expect similar results. However, a possible problem may arise from the interpretation of the questions: patients can answer differently only because they understand the questions in a different way.

In this issue of Respiration, Janssens et al. [8] present a study comprising patients suffering from chronic respiratory failure and treated by home mechanical ventilation using the French version of the MRF28 disease-specific health-related quality of life questionnaire. The study found a highly significant correlation with subscores of other validated questionnaires (e.g. the St. George's Respiratory Questionnaire, SF-36 and the Hospital Anxiety and Depression Scale) used for assessing QOL. As already cited in the references, the questionnaire has already been validated in clinical use, with interesting peculiarities which underline some of its original aspects. The current study confirms the already available results and contributes to the spread of this tool among French chest specialists.

I woud like to imagine a foreign country version of the cult movie 'Some like it hot', directed by Billy Wilder, with Marilyn Monroe and the characters' voices dubbed by foreign actors.

Perhaps, the French version of Marilyn's voice gives a different thrill!

\section{References}

1 Joint ACCP/AACVPR Evidence-Based Guidelines. Pulmonary Rehabilitation. Chest 1997; 112:1363-1396.

$>2$ De Blasio F: A Doubting Thomas dealing with pulmonary rehabilitation. Chest 2000;117: 929-931.

3 Carone M, Jones PW: Health status 'quality of life'. Eur Respir Mon 2000;13:22-35.
4 Guyatt GH, Berman LB, Townsend M, et al: A measure of quality of life for clinical trials in chronic lung disease. Thorax 1987;42:773778.

5 Jones PW, Quirk PF, Baveystock CM, et al: A self-complete measure for chronic airflow limitation - The St. George's Respiratory Questionnaire. Am Rev Respir Dis 1992;145:13211327.

6 Carone M, Bertolotti G, Anchisi F, et al: Analysis of factors that characterize health impairment in patients with chronic respiratory failure. Eur Respir J 1999;13:1293-1300.
7 Carone M, Donner CF, Jones PW, et al: The 28-item respiratory failure quality of life questionnaire (MRF28). Preliminary data from a multicenter, multinational study. Am J Respir Crit Care Med 1998;157:A116.

8 Janssens J.-P., Héritier-Praz A, Carone M, et al: Validity and reliability of a French version of the MRF-28 health-related quality of life questionnaire. Respiration 2004;71:567-573. 